\title{
ARCHETYPAL ANALYSIS: AN ALTERNATIVE TO CLUSTERING FOR UNSUPERVISED TEXTURE SEGMENTATION
}

\author{
ISMAEL CABERO ${ }^{1}$ AND IRENE EPIFANIO ${ }^{\bowtie, 2}$ \\ ${ }^{1}$ Department of Didactics of Mathematics, Universitat de València, Avda. Tarongers, 4, 46022 València, Spain; \\ ${ }^{2}$ Department of Mathematics and IMAC, Universitat Jaume I, Campus del Riu Sec, Castelló, 12071, Spain \\ e-mail: ismael.cabero@uv.es, epifanio@uji.es \\ (Received November 8, 2018; revised April 26, 2019; accepted April 30, 2019)
}

\begin{abstract}
Texture segmentation is one of the main tasks in image applications, specifically in remote sensing, where the objective is to segment high-resolution images of natural landscapes into different cover types. Often the focus is on the selection of discriminant textural features, and although these are really fundamental, there is another part of the process that is also influential, partitioning different homogeneous textures into groups. A methodology based on archetype analysis (AA) of the local textural measurements is proposed. AA seeks the purest textures in the image and it can find the borders between pure textures, as those regions composed of mixtures of several archetypes. The proposed procedure has been tested on a remote sensing image application with local granulometries, providing promising results.
\end{abstract}

Keywords: archetype, image segmentation, local granulometries, mathematical morphology, texture analysis.

\section{INTRODUCTION}

Image segmentation, and texture segmentation in particular, is one of the most important and difficult tasks in image processing. It consists of separating the different textures presented in the image. In this work, we focus on unsupervised texture segmentation, i.e., when no previous information about the textures in the image is available. Texture segmentation is part of the broader field of texture analysis (Tuceryan and Jain, 1993). Although the concept of texture has no exact definition in image processing, the underlying idea is that it is something where local patterns are repeated.

Two general approaches for carrying out texture segmentation are region-based approaches or boundary-based approaches. We consider a common region-based approach that consists of computing (local) textural features in small windows centered on each pixel of the image or on a sample of pixels and then performing a clustering analysis of them (Soille, 2003, Ch. 11). The reason for using clustering techniques is that feature vectors that have common attributes will form clusters in the feature space (Fletcher and Evans, 2005). However, this rationale does not take into account the cases in which the window is centered on the border between two or more different textures, i.e., if the window contains a mixture of textures.

In summary, that procedure returns a sample of features from different pure types of textures (when the windows contain a single kind of texture), but also a sample of features from a mixture of textures. For that reason, we propose a more appropriate unsupervised statistical learning technique as an alternative to cluster analysis: archetype analysis (AA). The objective of AA is precisely to extract the archetypes, which are pure profiles in a data set, and to express the data as mixtures of those archetypes. The archetypes are themselves a mixture of observations from the data set. Therefore, with AA we can obtain the set of pure textures in the image and find the borders between pure textures, as those regions that are composed of mixtures of several archetypes. Textures are described by features. Obtaining good results will depend on the following premises: using textural features that on the one hand can successfully separate the characteristics of the different pure textures in the image, and on the other hand, in the case of a mixture of textures, its features are a convex combination of the features of the pure textures that form the mixture.

A toy example is used to illustrate what AA means and how it differs from clustering. Let us assume that we have in Fig. 1 a sample of six small windows from a certain image to segment. In this sample, the first and last window contain pure textures; the first one is completely smooth, while the last one is noisy. However, the other windows are a mixture (in different degrees) of the two textures. In order to characterize the windows, we consider the following feature: the percentage of smoothness in the window, measured as the area of smooth zones in the window divided by the total area of the window. So, we can summarize each windows as 100, 80, 60, 40, 20 and 0, respectively. Applying $k$-means, with $k=2$, to this sample returns 80 
and 20 as centers, corresponding to the two windows in the central column of Fig. 1, which are mixtures. Applying AA (with two archetypes) to this sample instead returns 100 and 0 as archetypes, corresponding to the first and last window, which are pure textures. Clustering returns central points, while AA returns extreme points, which are purer profiles than the central points. Furthermore, in a matrix $\alpha$ AA returns the composition (from 0 to 1 and adding up to 1) of the windows as a function of the archetypes found. In this example, $\alpha$ is [ $10 ; 0.80 .2 ; 0.60 .4 ; 0.40 .6$; $0.20 .8 ; 0$ 1], i.e., it returns how archetypes are mixed in each sample. So through $\alpha$ we can know that the first and last window correspond to pure textures, each corresponding to a different archetype, while the other windows are mixtures, to some extent, of those two textures. For example, the second window is a mixture between $80 \%$ of the first archetype (the smooth one) and $20 \%$ of the second archetype (the noisy one).

AA was proposed by Cutler and Breiman (1994) and it has found applications in diverse fields, such as computer vision (Chen et al., 2014; Bauckhage et al., 2015; Sun et al., 2017a;b; Mair et al., 2017), developmental psychology (Ragozini et al., 2017), engineering (Epifanio et al., 2013; Vinué et al., 2015; Vinué, 2017; Epifanio et al., 2018b; MillánRoures et al., 2018), finance (Moliner and Epifanio, 2019), genetics (Thøgersen et al., 2013), global development (Epifanio, 2016; Epifanio et al., 2018a), machine learning problems (Mørup and Hansen, 2012), neuroscience (Tsanousa et al., 2015; Hinrich et al., 2016) and sports (Eugster, 2012; Vinué and Epifanio, 2017).

The purpose of this work is to introduce the idea of using AA for texture segmentation and to apply it to a real problem. Section "Material and methods" presents the data and describes the methodology. Section "Results" shows the segmentation results. Finally, conclusions and further developments are discussed in Section "Discussion". The code in R
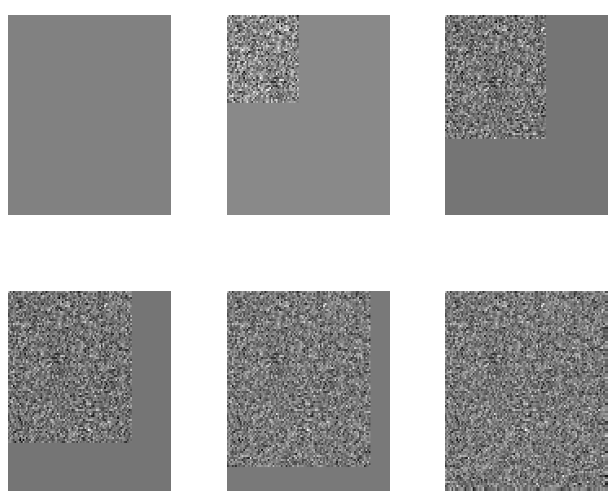

Fig. 1. Toy example illustrating the difference between clustering and AA results (see text for details).
(R Development Core Team, 2018) and data for reproducing the results are available at http://www3. uji.es/ epifanio/RESEARCH/aasegmentation.rar.

\section{MATERIAL AND METHODS}

\section{PRELIMINARY DEFINITIONS}

Let us review some basic morphological transformations (see Soille, 2003 for an in-depth introduction). Let $B$ be a structuring element, let $\breve{B}$ be the reflection of $B$, and let $f$ be a gray scale image. Then, the erosion of $f$ by $B$ is $\left[\varepsilon_{B}(f)\right](x)=$ $\min _{b \in B} f(x+b)$; the dilation of $f$ by $B$ is $\left[\delta_{B}(f)\right](x)=$ $\max _{b \in B} f(x+b)$, and the opening of $f$ by $B: \gamma_{B}(f)=$ $\delta_{\check{B}}\left[\varepsilon_{B}(f)\right]$.

Matheron (1975) defined a granulometry on a family $\mathscr{A}$ of sets, as a one-parameter family $\psi_{\lambda}$, with $\lambda \geq 0$, of mappings from $\mathscr{A}$ into itself such that: (i) $\psi_{\lambda}(A) \subset A$ for any $\lambda>0$ and $A \in \mathscr{A}$; (ii) if $A, B \in$ $\mathscr{A}$ and $A \subset B$, imply $\psi_{\lambda}(A) \subset \psi_{\lambda}(B)$; (iii) $\lambda_{1} \geq \lambda_{2}>$ 0 imply $\psi_{\lambda_{1}}(A) \subset \psi_{\lambda_{2}}(A)$ and (iv) $\psi_{\lambda_{1}} \circ \psi_{\lambda_{2}}=\psi_{\lambda_{2}} \circ$ $\psi_{\lambda_{1}}=\psi_{\text {sup }}\left(\lambda_{1}, \lambda_{2}\right)$. Additionally, it is usual to consider that $\psi_{\lambda}(A)=A$.

\section{DATA}

Compositions of artificial textures are the data sets commonly used for assessing texture segmentation procedures. However, we use several images of forest stands, with natural (real) textures, since textures on natural landscapes are more difficult to process owing to their high natural variation (Epifanio and Soille, 2007).

Many different local textural features can be used, such as classical spatial moments (Tuceryan, 1994) or Gabor filters (Jain and Farrokhnia, 1991), where features are computed in a sample of the pixels for computational efficiency and clustered.

Here, we prefer to use features based on mathematical morphology tools that have been proven to be successful in tackling different problems of geoscience and remote sensing (Epifanio and Ayala, 2002; Soille and Pesaresi, 2002; Plaza et al., 2005; Benediktsson et al., 2005; Fauvel et al., 2008). In particular, we use the well-known local granulometries (Dougherty et al., 1989), specifically granulometries by opening using squares of increasing size, as presented in (Soille, 2003, Ch. 11) for similar satellite images. Openings using squares $(S)$ of increasing size $\lambda$ obey the above definition. So, in our application we define a granulometric size distribution on $f$ by: $1-V\left(\gamma_{S}(f)\right) / V(f)$, where $V$ stands for the volume, i.e., the sum of the pixel values. 


\section{METHODOLOGY}

The proposed procedure uses two unsupervised learning procedures: trimmed $k$-means (CuestaAlbertos et al., 1997) and AA. First, these procedures are reviewed.

\section{Trimmed $k$-means}

Trimmed $k$-means is analogous to $k$-means but a proportion $\delta$ (between 0 and 1) of observations is discarded by the procedure itself, i.e., the trimmed points are self-determined by the data. Trimmed $k$ means aims to robustify $k$-means, i.e., to determine appropriate clusters when noisy data or outliers are present, which are detected and returned as trimmed observations by the procedure itself.

Let $x_{1}, \ldots, x_{n}$ be $n$ points of dimension $p$. Let $k$ be the number of clusters. The $k$-means returns a set of $k$ points, $m_{1}^{*}, \ldots, m_{k}^{*}$, the centroids, verifying $(\|\cdot\|$ denotes the Euclidean norm for vectors)

$$
\left\{m_{1}^{*}, \ldots, m_{k}^{*}\right\}=\arg \min _{m_{1}, \ldots, m_{k}} \frac{1}{n} \sum_{i=1}^{n} \inf _{1 \leq j \leq k}\left\|x_{i}-m_{j}\right\|^{2}
$$

and each observation $x_{i}$ is assigned to its closest centroid $m_{j}^{*}$.

The trimmed $k$-means, with trimming size $\delta$, returns $k$ points, $m_{1}^{*}, \ldots, m_{k}^{*}$ such that

$$
\begin{aligned}
& \left\{m_{1}^{*}, \ldots, m_{k}^{*}\right\}= \\
& \arg \min _{\mathbf{Y},\left\{m_{1}, \ldots, m_{k}\right\}} \frac{1}{\lceil n(1-\delta)\rceil} \sum_{x_{i} \in \mathbf{Y}} \inf _{1 \leq j \leq k}\left\|x_{i}-m_{j}\right\|^{2},
\end{aligned}
$$

where $\mathbf{Y}$ ranges on subsets of $x_{1}, \ldots, x_{n}$ containing $\lceil n(1-\delta)\rceil$ points $(\lceil\cdot\rceil$ denotes the integer part of a given value). Each non-trimmed point $x_{i}$ is assigned to its closest centroid $m_{j}$. A trimmed $k$-means algorithm can be found in García-Escudero et al. (2003).

\section{Archetype analysis}

In AA, three matrices are returned: 1) the $k$ archetypes $\mathbf{z}_{j}$, which are the rows of a $k \times p$ matrix $\mathbf{Z} ; 2)$ an $n \times k$ matrix $\alpha=\left(\alpha_{i j}\right)$ that contains the mixture coefficients that approximate each observation $\mathbf{x}_{i}$ by a mixture of the archetypes $\left(\hat{\mathbf{x}}_{i}=\sum_{j=1}^{k} \alpha_{i j} \mathbf{z}_{j}\right)$; and 3) a $k \times n$ matrix $\beta=\left(\beta_{j l}\right)$ that contains the mixture coefficients that define each archetype $\left(\mathbf{z}_{j}=\right.$ $\left.\sum_{l=1}^{n} \beta_{j l} \mathbf{x}_{l}\right)$. To find these matrices, we minimize the following residual sum of squares (RSS):

$$
\begin{aligned}
\mathrm{RSS} & =\sum_{i=1}^{n}\left\|\mathbf{x}_{i}-\sum_{j=1}^{k} \alpha_{i j} \mathbf{z}_{j}\right\|^{2} \\
& =\sum_{i=1}^{n}\left\|\mathbf{x}_{i}-\sum_{j=1}^{k} \alpha_{i j} \sum_{l=1}^{n} \beta_{j l} \mathbf{x}_{l}\right\|^{2},
\end{aligned}
$$

under the constraints

1) $\sum_{j=1}^{k} \alpha_{i j}=1$ with $\alpha_{i j} \geq 0$ for $i=1, \ldots, n$ and

2) $\sum_{l=1}^{n} \beta_{j l}=1$ with $\beta_{j l} \geq 0$ for $j=1, \ldots, k$.

To estimate those matrices Cutler and Breiman (1994) developed an alternating minimizing algorithm, which was implemented in R by Eugster and Leisch (2009).

If we know that the number of textures on the image is $k$, we can use this number for the AA algorithm. Otherwise, a simple but effective heuristic (Cutler and Breiman, 1994; Eugster and Leisch, 2009; Vinué et al., 2015; Seth and Eugster, 2016) such as the elbow criterion can be used. With the elbow criterion, we plot the RSS for different $k$ values and the value of $k$ is selected as the point where the elbow is located.

\section{Proposed procedure}

The idea of the procedure is to extract textural features in small windows around each pixel of the image to segment. These textural features describe the windows. Then, we look for the extreme or archetypal features that correspond to the pure textures (AA is used). The textural features of the windows are expressed as a convex combination (the $\alpha$ matrix) of the archetypal features. One of the $\alpha$ values will be high (near one) for the windows with pure or nearly pure textures. However, windows with mixed textures because they are on the border between different textures will have intermediate $\alpha$ values. We can cluster the textural features using the $\alpha$ values to segment the image. If we assume that the percentage of windows with mixed textures because they are on the border between different textures is small, those windows would correspond to the trimmed observations of the trimmed $k$-means. Let us look at an implementation of this idea based on the description of textures by granulometric curves.

Our texture segmentation algorithm consists of the following steps: (a) Computing the granulometric curves within a small window around each pixel, (b) performing $\mathrm{AA}$ on the granulometric curves of a sample of pixels in the image and selecting a certain number $k$ of archetypes by using the elbow criterion, (c) performing a trimmed $k$-means of the $\alpha$ values and (d) classifying every pixel in the image according to the results of step (c) by computing the $\alpha$ values corresponding to the archetypes obtained in step (b). 
The granulometric curves are not part of the methodology and could be substituted for other appropriate textural features. Note that if the scales of new textural features are not comparable, they should be standardized before applying AA.

The selection of the window size depends on the image resolution, which should be high enough to capture the textures.

\section{RESULTS}

First the proposed procedure is illustrated by an image of several forests and compared with alternative unsupervised learning methods other than AA. In Sect. 3.1 the methodology is applied in a remote sensing problem and compared with other methodologies.

Fig. 2a shows the image to segment. We have computed the granulometric curves by opening using squares of increasing size (from 1 to 50 ), in windows with a size of $51 \times 51$ centered on a systematic sample of pixels, as shown in Fig. 2b, where each color indicates a different texture and white indicates that the pixel is on a border between textures. Those labels will act as a ground truth. The 294 granulometries are shown in Fig. 3. The distribution functions for the group colored in cyan move from 0 to 1 more rapidly (i.e., they have a more concentrated probability distribution) than the corresponding ones from the group colored in red, and these move more rapidly than the distribution functions from the class colored in yellow. This occurs because the grain sizes (tree canopy) are small for the cyan class and they increase for the red and yellow classes.

AA is applied to the granulometries from $k=1$ to 10. Fig. 4 shows the screeplot (RSS versus the number of archetypes). According to the elbow criterion, $k$ $=3$ is selected, which is the number of true texture classes, although it is determined in an unsupervised way. The archetypes (together with the $\alpha$ values) are computed for $k=3$. The nearest granulometric curves to each archetype are the curves from the samples with numbers 28, 99 and 190. The windows surrounding those archetypal pixels are displayed in Fig. 5. In this way, we obtain a (pure) representative of each class.

The ternary plot with the $\alpha$ values is shown in Fig. 6. The different colors and numbers represent true classes, as in Fig. 2b, where the black points with the number 0 indicate the pixels on borders between textures. Note that the majority of points are situated around the archetypes, each of which is on one corner of the plot. In the middle of the plot there are some spread points, which are a mixture of the archetypes,

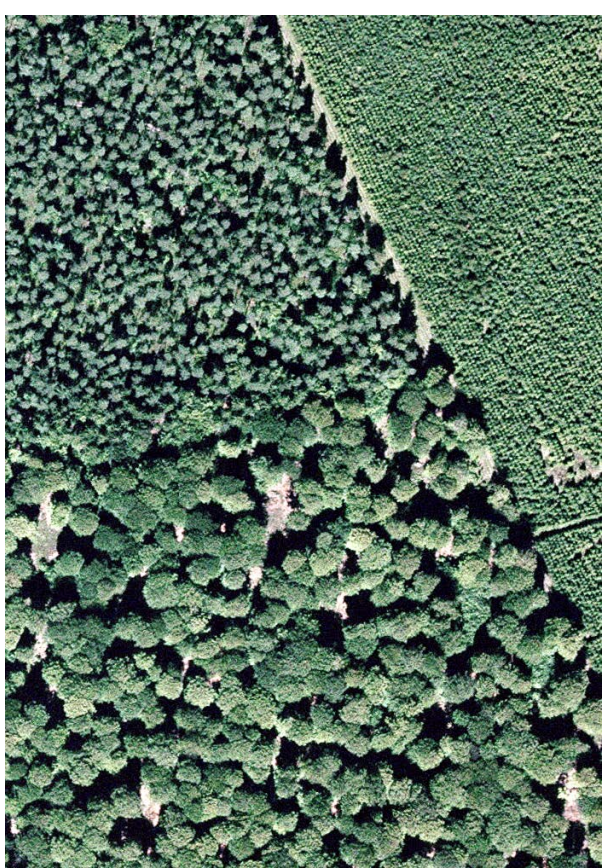

(a)

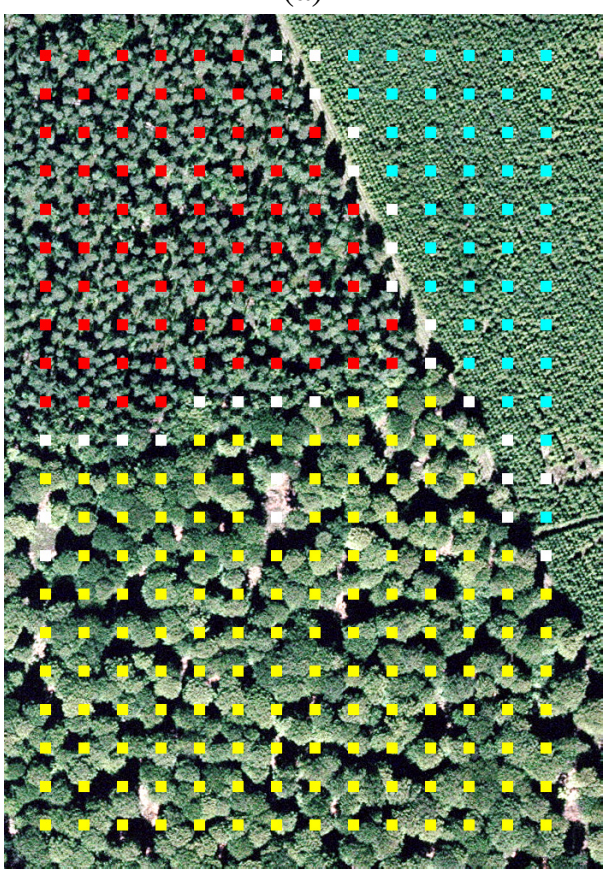

(b)

Fig. 2. (a) Image of several forests. (b) Sampled pixels with their respective true label (see text for details).

and the majority of them are shown in black coded with zero, i.e., they are border pixels. Therefore, our premises are met. We apply the trimmed $k$-means, with $k=3$ and the proportion of trimmed sample $\delta=0.095$, the percentage of pixels coded with zero, which can be considered as outliers. The matching matrix is shown in Table 1. The errors occur in border pixel classes. We have computed the adjusted Rand index (Hubert and Arabie, 1985) to compare the true partition 


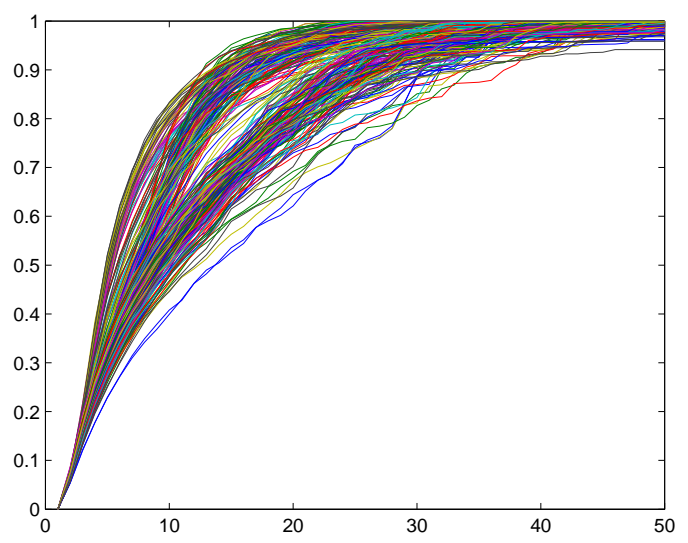

Fig. 3. Granulometric curves.

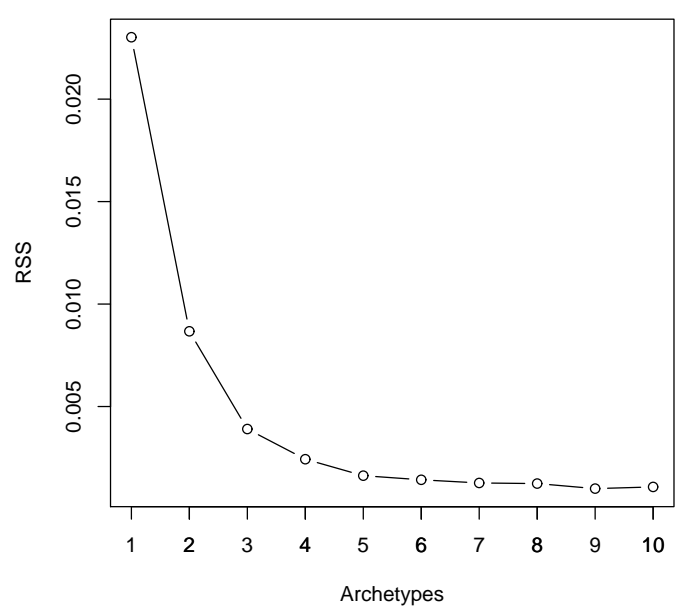

Fig. 4. Screeplot of the residual sum of squares.
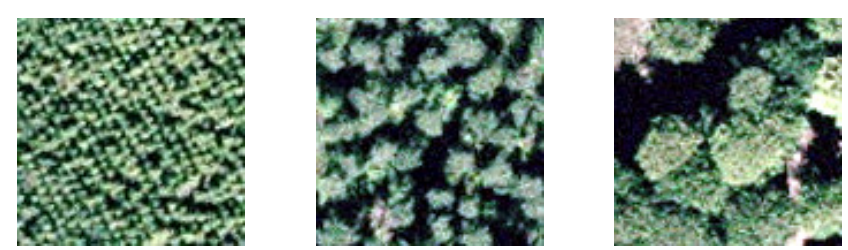

Fig. 5. Archetypal representative of each group.

with the one obtained using our methodology. The Adjusted Rand Index (ARI) is 0.87, which indicates a high level of agreement between the partitions (the closer to one, the more similar the partitions are).

We have also computed the ARI using different $\delta$ values in order to check the robustness of the results when the exact percentage of border pixels is not known. When $\delta=0$, the classical $k$-means is applied. Furthermore, in order to test the improvement achieved by using AA instead of a clustering algorithm in step (b), for comparative purposes we change AA in step (b) for one of two possibilities. The first possibility, referred to as clustering, consists of applying the clustering algorithm ( $k$-means or its trimmed version) directly to the granulometries. The second possibility, referred to as PC clustering, consists of applying the clustering algorithm ( $k$-means or its trimmed version) to the principal component (PC) scores of the granulometries. The first two PCs are considered, since they explain more than $95 \%$ of the variability and better results are obtained than if we consider, for example, the first five PCs, which explain more than $99 \%$ of the variability. Table 2 shows the ARI values. On the one hand, for all the $\delta$ values, the highest ARI is obtained by AA, showing that AA returns the most similar the partition to the ground truth. On the other hand, for all the $\delta$ values, the ARI values for AA are high, in the majority of cases above 0.85 .

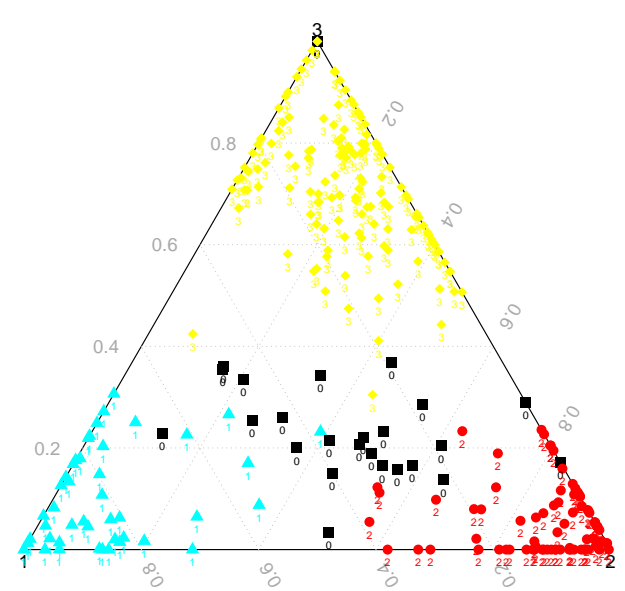

Fig. 6. Ternary plot (see text for details about the color and number codes).

Table 1. Matching matrix on the sampled pixels (true class labels in the first row; labels obtained with out methodology in the first column).

\begin{tabular}{ccccc}
\hline & 0 & 1 & 2 & 3 \\
\hline 0 & 17 & 3 & 1 & 6 \\
1 & 6 & 77 & 0 & 0 \\
2 & 4 & 0 & 136 & 0 \\
3 & 1 & 0 & 0 & 43 \\
\hline
\end{tabular}

\section{APPLICATION}

We consider the segmentation of orthophotos. Fig. 7 shows two $50 \mathrm{~cm}$ resolution images of the Alps used to assess the accuracy of a method monitoring the increase in woody vegetation from multitemporal Landsat images (Maggi et al., 2007). These orthophotos need to be segmented into three different classes of tree densities: dense, sparse, and empty. 

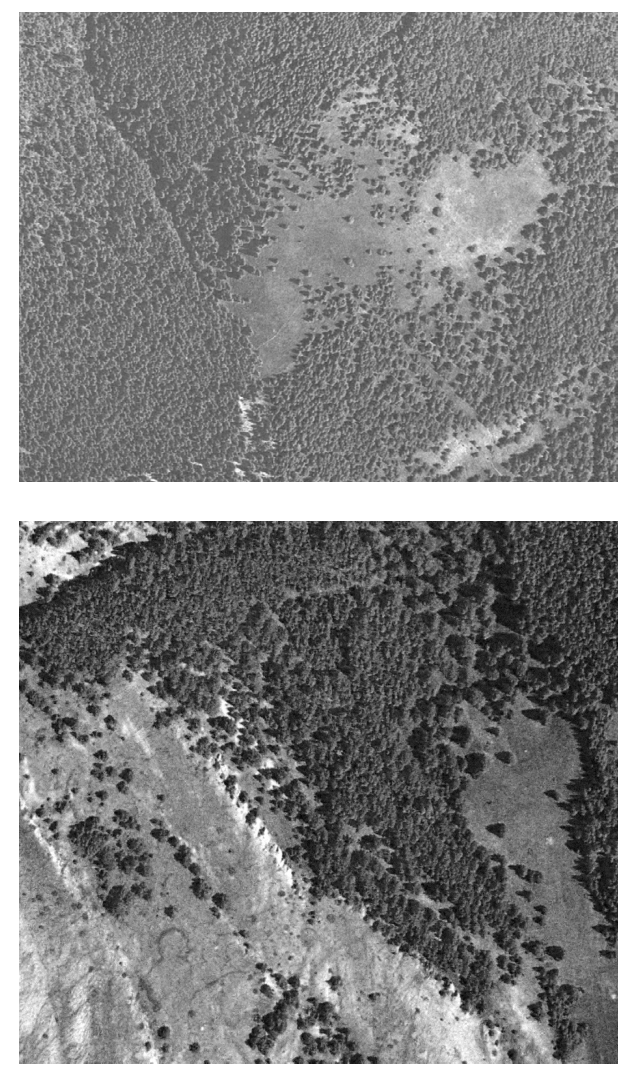

Fig. 7. Orthophotos with dense, sparse, and empty tree densities.

These orthophotos were originally used in Epifanio and Soille (2007) with a supervised texture segmentation methodology. This consisted of describing each pixel by several morphological features computed in a centered window of size 81 , manually selecting nine prototypes from an orthophoto as the training data (five for dense, three for sparse, and one for the empty class), and using the minimum distance classifier, i.e., the nearest neighbor according to the Euclidean distance. The segmentation results with this supervised methodology are shown in Fig. 8. The boundaries of the dense vegetation zones and the treeless zones are traced in white and black, respectively. The remaining areas correspond to the zones with sparse tree density. In Supplementary Material the labeled regions are visualized.

We now apply the approach described in Jain and Farrokhnia (1991) using Gabor filters to perform unsupervised texture segmentation, as explained in MathWorks (2019). A total of 32 Gabor features and
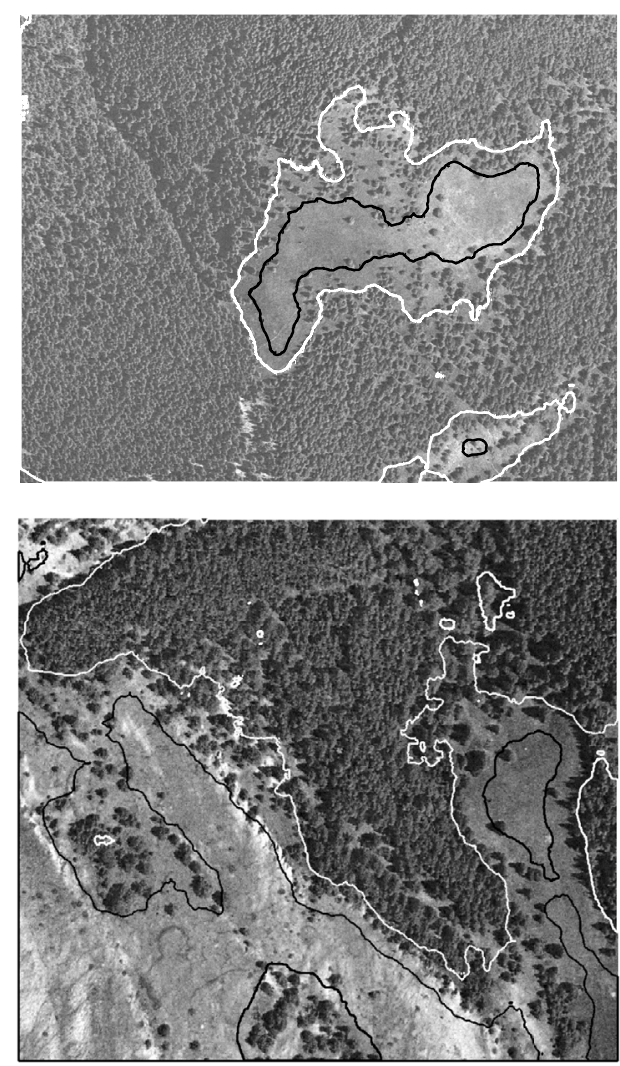

Fig. 8. Supervised segmentation of orthophotos with the methodology described in Epifanio and Soille (2007).

2 spatial features for each pixel in the input image are clustered with $k$-means. Fig. 9 shows the segmentation. Boundaries traced in white and black for the first orthophoto separate the classes returned. The obtained segmentation does not correspond to the density of trees. Even if we consider two or four groups instead three classes, the segmentation does not correspond to the density of trees (Fig. 10). For the second orthophoto, as before, the boundaries of the dense vegetation zones and the treeless zones are traced in white and black, respectively. There are several clear errors in this segmentation, for example, in the top left part of the image a dense vegetation zone is segmented as sparse; and a zone in the bottom right of the image with vegetation is classified as empty. In Supplementary Material the labeled regions are visualized.

We now apply our proposed procedure with windows of size 81 . We apply AA and $\delta=0$ for the trimmed $k$-means to facilitate comparison with the 

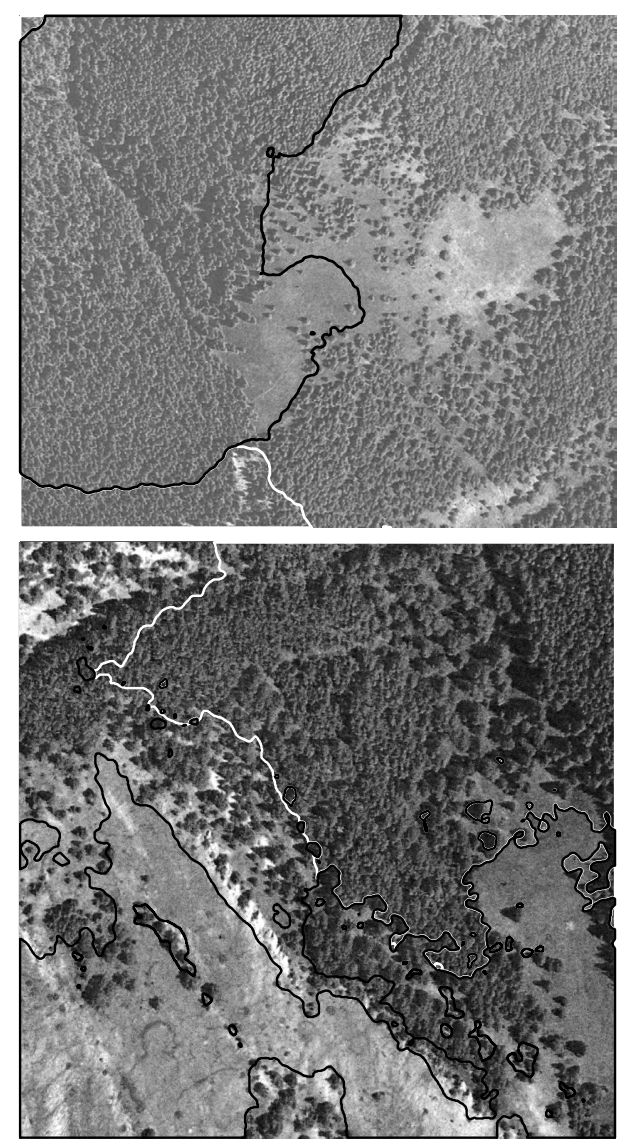

Fig. 9. Unsupervised segmentation of orthophotos using Gabor filters.

Table 2. ARI for clustering, PC clustering and AA, using different $\delta$ values.

\begin{tabular}{cccc}
\hline$\delta$ & Clustering & PC clustering & AA \\
\hline 0.00 & 0.82 & 0.82 & 0.85 \\
0.01 & 0.83 & 0.83 & 0.85 \\
0.02 & 0.83 & 0.83 & 0.86 \\
0.03 & 0.81 & 0.82 & 0.88 \\
0.04 & 0.81 & 0.81 & 0.88 \\
0.05 & 0.80 & 0.80 & 0.89 \\
0.06 & 0.78 & 0.81 & 0.88 \\
0.07 & 0.79 & 0.81 & 0.88 \\
0.08 & 0.78 & 0.79 & 0.87 \\
0.09 & 0.77 & 0.78 & 0.86 \\
0.10 & 0.76 & 0.78 & 0.87 \\
0.11 & 0.75 & 0.77 & 0.86 \\
0.12 & 0.74 & 0.76 & 0.83 \\
0.13 & 0.73 & 0.74 & 0.82 \\
0.14 & 0.72 & 0.73 & 0.80 \\
0.15 & 0.71 & 0.72 & 0.79 \\
\hline
\end{tabular}
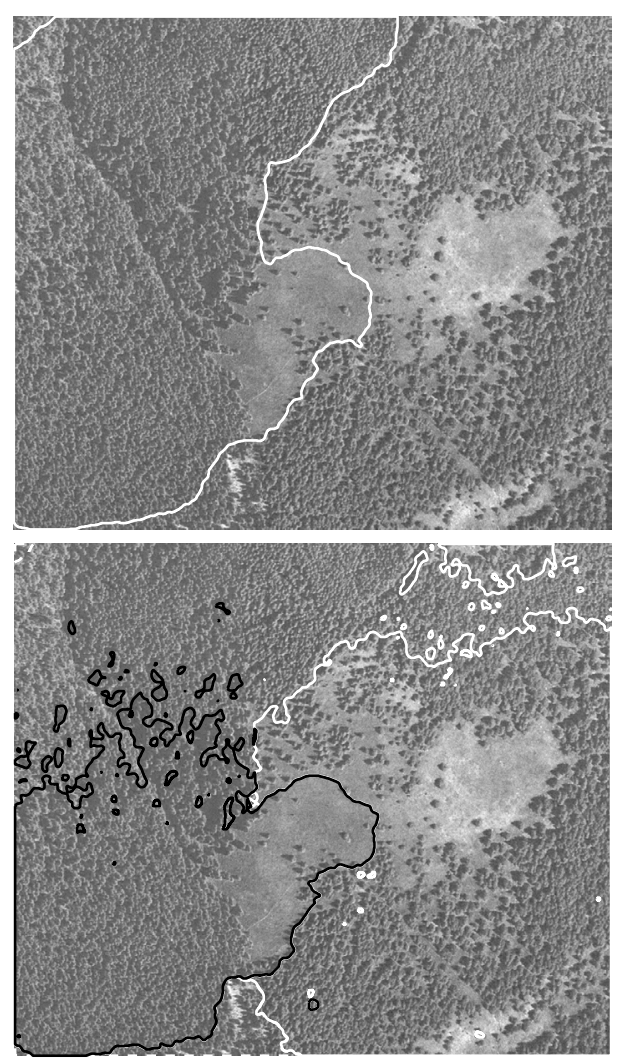

Fig. 10. Unsupervised segmentation of the first orthophoto using Gabor filters with two and four classes.

other methods, i.e., no observation is trimmed. For the first orthophoto the elbow is at $k=4$, so for the first orthophoto we consider four classes. The boundaries of the dense vegetation zones are traced in white (the areas surrounded by dashed lines have lower density than the solid lines).

The boundaries of the treeless zones are traced in black, and the remaining areas correspond to the zones with sparse tree density. Fig. 11 shows the segmentation results with our proposed procedure. If instead of using AA, the local granulometries are directly clustered by $k$-means, the segmentation obtained can be seen in Fig. 12. In this last case, some dense vegetation zones are misclassified as sparse tree density zones. In Supplementary Material the labeled regions are visualized.

Our procedure returns very satisfactory segmentation results, comparable with the results of the supervised methodology, despite being completely unsupervised, i.e., no information is provided. 

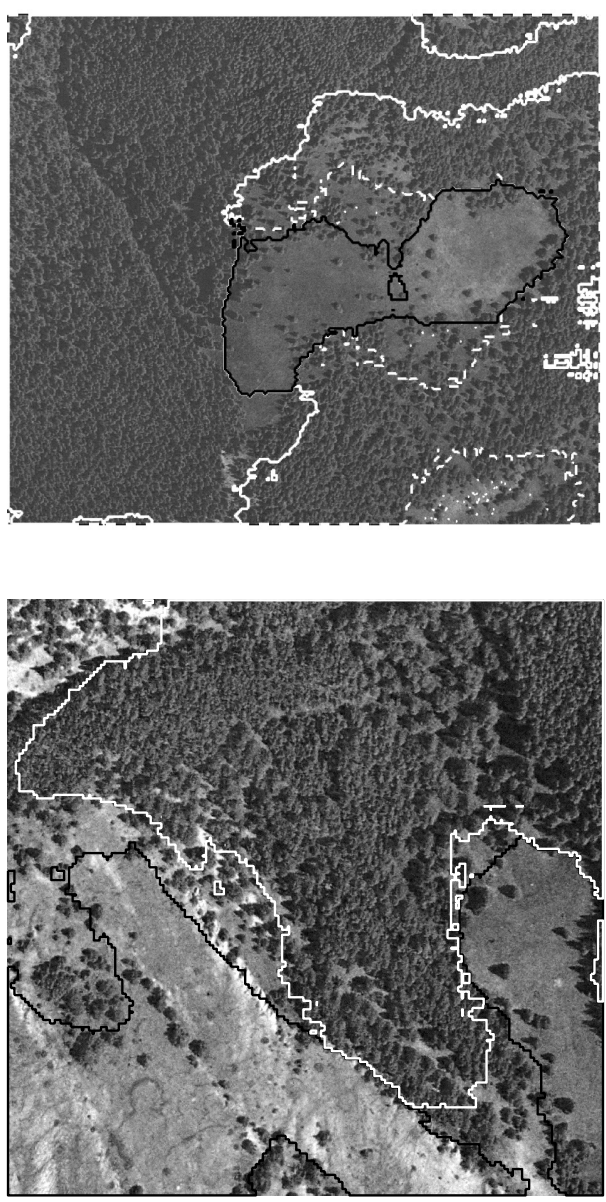

Fig. 11. Unsupervised segmentation of orthophotos using our procedure.

\section{DISCUSSION}

A common problem of texture segmentation is that local windows centered on each pixel from which features are extracted can contain more than one texture. Some attempts to solve this consist of considering windows not centered on the pixel, as in Wang et al. (1993) and Epifanio and Soille (2007). However, those procedures are more laborintensive than the procedure that we propose, where windows are centered on each pixel, and what we change is the clustering phase for an archetypal analysis phase followed by a clustering phase. The preliminary results show the relevance of the proposed approach. Our procedure has been compared with other methods. In spite of its simplicity, the relevance of its results has been shown, especially in a remote sensing application. The results of our procedure are competitive not only with other unsupervised methodologies, but also in comparison with a supervised methodology.
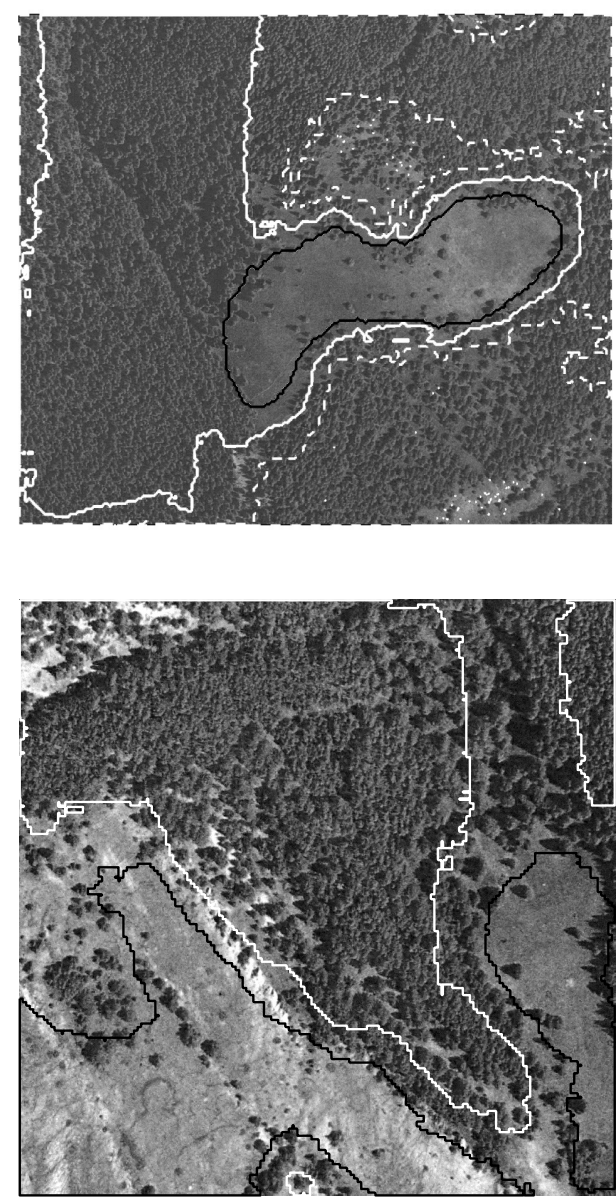

Fig. 12. Unsupervised segmentation of orthophotos by clustering the local granulometries.

Our procedure can also return archetypal windows of each group. Instead of using the $\alpha$ values in the segmentation, the archetypal windows themselves could be used in the segmentation process by acting as prototypes.

Some additional points could also be studied. As an alternative to AA, we could use archetypoid analysis (ADA) (Vinué et al., 2015), where instead of the archetypal representatives being built as a mixture of observations, they are actual observations. Simmilarly, instead of trimmed $k$-means, we could use trimmed $k$-medoids (Ibáñez et al., 2012). On the other hand, we have treated textural features as multivariate features, since this is the most usual case in this context and we have preferred to do this due to comparison with other well-known techniques. We have not exploited the fact that granulometries are functions, and functional data analysis (Ramsay and Silverman, 2005) techniques could be used; in fact, functional archetypal analysis (Epifanio, 2016) could have been used. 
For other applications, alternative textural features could be considered, since the proposed methodology does not depend on the selected textural features, but rather on the fact that they are discriminant between classes and the mixtures of textures are transferred to the vector of characteristics.

\section{ACKNOWLEDGMENTS}

This work has been partially supported by Grant DPI2017-87333-R from the Spanish Ministerio de Ciencia, Innovación y Universidades (AEI/FEDER, UE) and UJIB2017-13 from Universitat Jaume I. We would also like to thank Pierre Soille for providing us with the images.

\section{REFERENCES}

Bauckhage C, Kersting K, Hoppe F, Thurau C (2015). Archetypal Analysis as an Autoencoder. In: Workshop New Challenges in Neural Computation.

Benediktsson J, Palmason J, Sveinsson J (2005). Classification of hyperspectral data from urban areas based on extended morphological profiles. IEEE Geosci Remote 43:480-91.

Chen Y, Mairal J, Harchaoui Z (2014). Fast and Robust Archetypal Analysis for Representation Learning. In: Proc 2014 IEEE Conf Comput Vision Pattern Recong (CVPR) 1478-85.

Cuesta-Albertos JA, Gordaliza A, Matrán C (1997). Trimmed $k$-means: an attempt to robustify quantizers. Ann Stat 25:553-76.

Cutler A, Breiman L (1994). Archetypal Analysis. Technometrics 36:338-47.

Dougherty ER, Kraus EJ, Pelz JB (1989). Image segmentation by local morphological granulometries. In: Proc 12th Can Symp Remote Sens Geosci Remote Sens Symp 3:1220-3.

Epifanio I (2016). Functional archetype and archetypoid analysis. Comput Stat Data An 104:24-34.

Epifanio I, Ayala G (2002). A random set view of texture classification. IEEE T Image Process 11:859-67.

Epifanio I, Ibáñez MV, Simó A (2018a). Archetypal analysis with missing data: see all samples by looking at a few based on extreme profiles. Am Stat, in press.

Epifanio I, Ibáñez MV, Simó A (2018b). Archetypal shapes based on landmarks and extension to handle missing data. Adv Data Anal Classi 12:705-35.

Epifanio I, Soille P (2007). Morphological texture features for unsupervised and supervised segmentations of natural landscapes. IEEE Geosci Remote 45:1074-83.

Epifanio I, Vinué G, Alemany S (2013). Archetypal analysis: contributions for estimating boundary cases in multivariate accommodation problem. Comput Ind Eng 64:757-65.

Eugster MJ, Leisch F (2009). From Spider-Man to Hero Archetypal Analysis in R. J Stat Soft 30:1-23.

Eugster MJA (2012). Performance profiles based on archetypal athletes. Int J Perf Anal Spor 12:166-87.

Fauvel M, Benediktsson J, Chanussot J, Sveinsson J (2008). Spectral and spatial classification of hyperspectral data using SVMs and morphological profiles. IEEE Geosci Remote 46:3804-14.

Fletcher ND, Evans AN (2005). Texture segmentation using area morphology local granulometries. In: Ronse C, Najman L, Decencière E, eds., Mathematical Morphology: 40 Years On. Dordrecht: Springer.

García-Escudero LA, Gordaliza A, Matrán C (2003). Trimming tools in exploratory data analysis. J Comput Graph Stat 12:434-49.

Hinrich JL, Bardenfleth SE, Roge RE, Churchill NW, Madsen KH, Mørup M (2016). Archetypal analysis for modeling multisubject fMRI data. IEEE J Sel Top Signa 10:1160-71.

Hubert L, Arabie P (1985). Comparing partitions. J Classif 2:193-218.

Ibáñez MV, Vinué G, Alemany S, Simó A, Epifanio I, Domingo J, Ayala G (2012). Apparel sizing using trimmed PAM and OWA operators. Expert Syst Appl 39:10512-20.

Jain AK, Farrokhnia F (1991). Unsupervised texture segmentation using Gabor filters. Pattern Recogn 24:1167-86.

Maggi M, Estreguil C, Soille P (2007). Woody vegetation increase in alpine areas: a proposal for a classification and validation scheme. Int J Remote Sens 28:143-66.

Mair S, Boubekki A, Brefeld U (2017). Frame-based data factorizations. In: Proc 34th Int Conf Machine Learn (ICML'17) 70:2305-13.

Matheron G (1975). Random sets and integral geometry. New York: Wiley.

MathWorks (2019). Texture segmentation using Gabor filters. Accessed 2019-04-05.

Millán-Roures L, Epifanio I, Martínez V (2018). Detection of anomalies in water networks by functional data analysis. Math Probl Eng 2018:5129735

Moliner J, Epifanio I (2019). Robust multivariate and functional archetypal analysis with application to financial time series analysis. Physica A 519:195-208.

Mørup M, Hansen LK (2012). Archetypal analysis for machine learning and data mining. Neurocomputing 80:54-63.

Plaza A, Martinez P, Plaza J, Perez R (2005). Dimensionality reduction and classification of 
hyperspectral image data using sequences of extended morphological transformations. IEEE Geosci Remote 43:466-79.

R Development Core Team (2018). R: A language and environment for statistical computing. R Foundation for Statistical Computing, Vienna, Austria. ISBN 3900051-07-0.

Ragozini G, Palumbo F, D'Esposito MR (2017). Archetypal analysis for data-driven prototype identification. Stat Anal Data Min 10:6-20.

Ramsay JO, Silverman BW (2005). Functional data analysis, 2nd ed. New York: Springer.

Seth S, Eugster MJA (2016). Probabilistic archetypal analysis. Mach Learn 102:85-113.

Soille P (2003). Morphological image analysis: Principles and applications, 2nd ed. Berlin, Heidelberg: Springer.

Soille P, Pesaresi M (2002). Advances in mathematical morphology applied to geoscience and remote sensing. IEEE Geosci Remote 40:2042-55.

Sun W, Yang G, Wu K, Li W, Zhang D (2017a). Pure endmember extraction using robust kernel archetypoid analysis for hyperspectral imagery. ISPRS J Photogramm 131:147-59.

Sun W, Zhang D, Xu Y, Tian L, Yang G, Li W (2017b). A probabilistic weighted archetypal analysis method with Earth mover's distance for endmember extraction from hyperspectral imagery. Remote Sensing 9:841.

Thøgersen JC, Mørup M, Damkiær S, Molin S, Jelsbak L (2013). Archetypal analysis of diverse pseudomonas aeruginosa transcriptomes reveals adaptation in cystic fibrosis airways. BMC Bioinformatics 14:279.

Tsanousa A, Laskaris N, Angelis L (2015). A novel single-trial methodology for studying brain response variability based on archetypal analysis. Expert Syst Appl 42:8454-62.

Tuceryan M (1994). Moment-based texture segmentation. Pattern Recogn Lett 15:659-68.

Tuceryan M, Jain AK (1993). Texture analysis. In: Chen CH, Pau LF, Wang PSP, eds., Handbook of Pattern Recognition \& Computer Vision. River Edge: World Scientific, 235-76.

Vinué G (2017). Anthropometry: An R package for analysis of anthropometric data. J Stat Soft 77:1-39.

Vinué G, Epifanio I (2017). Archetypoid analysis for sports analytics. Data Min Knowl Disc 31:1643-77.

Vinué G, Epifanio I, Alemany S (2015). Archetypoids: A new approach to define representative archetypal data. Comput Stat Data An 87:102-15.

Wang D, Haese-Coat V, Bruno A, Ronsin J (1993). Texture classification and segmentation based on iterative morphological decomposition. J Vis Commun Image $\mathrm{R}$ $4: 197-214$. 